\title{
A tight correlation for GRB afterglows with canonicali light curves
}

Maria Giovanna Dainotti

Obserwatorium Astronomiczne, Uniwersytet Jagielloński, ul. Orla 171, 31-501 Kraków, Poland E-mail: mariagiovannadainotti@yahoo.it

\section{Vincenzo F. Cardone}

Dipartimento di Scienze Fisiche, Universit'a di Napoli ŞFederico II, ̌̌ Complesso Universitario di Monte Sant ̌̌Angelo, Edificio N, via Cinthia, 80126-Napoli, Italy

E-mail: winny@enodrac.gmail.com

\section{Salvatore Capozziello}

Dipartimento di Scienze Fisiche, Universit'a di Napoli ŞFederico II, ̌̌ Complesso Universitario di Monte Sant ̌̌Angelo, Edificio N, via Cinthia, 80126-Napoli, Italy

E-mail: capozziello@na.infn.it

\section{Michał Ostrowski}

Obserwatorium Astronomiczne, Uniwersytet Jagielloński, ul. Orla 171, 31-501 Kraków, Poland E-mail: miodoa.uj.edu.pl

\section{Richard Willingale}

Department of Physics \& Astronomy, University of Leicester, Road Leicester LE1 7RH, United Kingdom

E-mail:rw@star.le.ac.uk

Gamma -ray bursts (GRBs) observed up to redshifts $z>8$ are fascinating objects to study due to their still unexplained relativistic outburst mechanisms and a possible use to test cosmological models. Our analysis of 77 GRB afterglows with known redshifts revealed a physical subsample of long GRBs with canonical plateau breaking to power-law light curves with a significant luminosity $L_{X}^{*}$-break time $T_{a}^{*}$ correlation in the GRB rest frame. It proves that within the full sample of afterglows there exist physical subclasses revealed here by tight correlations of their afterglow properties. Following this analysis we extend it to correlations between the afterglow and the prompt emission GRB physical parameters. We reveal a tight physical scaling between the mentioned afterglow luminosity $L_{a}^{*}$ and the prompt emission mean luminosity $<L_{p}^{*}>_{45} \equiv E_{i s o} / T_{45}^{*}$, with the Spearman correlation coefficient reaching 0.95 for the data subsample with most regular light curves. We also analyzed correlations of $L_{a}^{*}$ with several other prompt emission parameters, including the isotropic energy $E_{i s o}$.

25th Texas Symposium on Relativistic Astrophysics - TEXAS 2010

December 06-10, 2010

Heidelberg, Germany 


\section{Introduction}

The detection of GRBs up to high redshifts ( $\mathrm{z}=8.2 ;[36,42])$, larger than Type I Supernovae Ia (SNeIa) $\left(z_{\max }=1.77\right.$; [35]), makes these objects appealing for possible use in cosmology. The problem is that GRBs seem not to be standard candles, with their energetics spanning over 7 orders of magnitude. Anyway, several GRB luminosity indicators [1, 18, 31, 22, 28, 29, 21, 33] and their use to constrain cosmological parameters $[19,30,41,26]$ have been proposed till now. Furthermore, [10] following [9] have derived an updated GRB Hubble diagram using the $\log L_{X}^{*}-\log T_{a}^{*}$ ("LT") ${ }^{1}$ correlation with five other two-dimensional GRB correlations used by [39]. However, the problem of large data scatters in the considered luminosity relations [5, 44] and a possible impact of detector thresholds on cosmological standard candles [38] have been discussed controversially [8]. Among these attempts, [12] have proposed a way to standardize GRBs as distance indicator with the discovery of the LT anti-correlation. The fitted power-law relation is $\log L_{X}^{*}=\log a+b \cdot \log T_{a}^{*}$; the constants $a$ and $b$ are determined using the [16] method. One may note that an analogous LT relation was derived phenomenologically by [23] and [25] and that the LT correlation is also a useful test for the models of [7] and [15].

We study the LT correlation using the extended GRB data set and demonstrate the existence of a physical LT scaling for "canonical" light curves in the GRB rest frame. Revealing these physical correlations can help the (still unclear) interpretation of the physical mechanisms responsible for the GRB X -ray afterglow emission and can infer important information about the nature of the emitting source. We also find that the prompt-afterglow correlations are more significant if one uses the prompt emission mean luminosity instead of the energy $E_{i s o}$. This work reveals an important fact: any search for physical relations between GRB properties should involve selection of well constrained physical GRB subsamples. Usage of all available data introduces into analysis the events with highly scattered intrinsic physical properties, what smooths out possible correlations, and may lead to systematic shifts of the fitted relations, e.g. [13]. It is likely that a substantial fraction of the observed large scatter is introduced because we are observing different classes of GRBs with different progenitors and/or in different physical conditions. Identifying such subclasses may be the real challenge. Separating short and long GRBs is too simplistic. Below, we demonstrate that a particular class of canonical GRBs exists within the full sample of long GRBs. In the paper we use CGS units: [erg] for energy, [erg/s] for luminosity and [s] for time. All quantities used for correlation analysis are computed in the GRB rest frames (we indicate such quantities using a superscript *, $E_{\text {iso }}$ is in GRB rest frame from its definition).

\section{Data selection and analysis}

We have analyzed a sample of all afterglows with known redshifts detected by $\underline{\text { Swift }}$ from 2005 January up to 2009 April, for which the light curves include early X-ray Telescope (XRT) data and therefore can be fitted by a Willingale's phenomenological model [43]. The redshifts $z$ are taken from the Greiner's Web site http://www.mpe.mpg.de/ jcg/grb.html in agreement with

\footnotetext{
${ }^{1}$ We use the index "*" to indicate quantities measured in the GRB rest frame in which $L_{X}^{*} \equiv L_{X}^{*}\left(T_{a}^{*}\right)$ is an isotropic $\mathrm{X}$-ray luminosity in the time $T_{a}^{*}$, the transition time separating the afterglow plateau and the power-law decay phases [12].
} 
the values reported by $[4,6]$. Our data analysis, including derivation of $T_{a}^{*}$ and $L_{X}^{*}$ (in units of (s) and $\left(\mathrm{ergs}^{-1}\right)$, respectively) for each afterglow, follows [12] and [43]. The source rest -frame

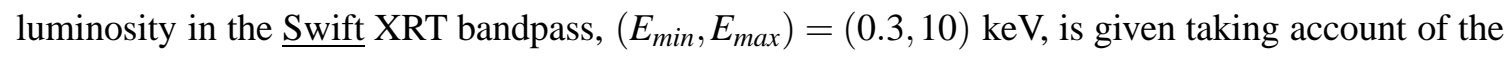
$K$-correction for cosmic expansion [3] by the following expression:

$$
L_{X}^{*}=\frac{4 \pi D_{L}^{2}(z) F_{X}}{(1+z)^{1-\beta_{a}}}
$$

where $F_{X}=F_{a} \exp \left(-\frac{T_{p}}{T_{a}}\right)$ is the observed flux $\left(\mathrm{erg} / \mathrm{cm}^{2} / \mathrm{s}\right)$ at the time $T_{a}, D_{L}(z)$ is the GRB luminosity distance for the redshift $z$, computed assuming a flat $\Lambda \mathrm{CDM}$ cosmological model with $\Omega_{M}=0.291$ and $h=0.697$. We have derived a spectral index $\beta_{a}$ for each GRB afterglow using the Evan's Web site http://www.swift.ac.uk/xrt curves [17] setting a filter time as $T_{a} \pm \sigma_{T_{a}}$; the $T_{a}$ values together with their errorbars, $\sigma_{T_{a}}$, are derived in the fitting procedure used by [43].

A choice of the Willingale model to obtain the fitted parameters $F_{a}, T_{a}$ and $T_{p}$ as a representation for the X-ray GRB light curves allows us to use a homogeneous sample of events to study physical correlation in a statistical way. To analyze how the accuracy of fitting the canonical light curve to the data influences the studied correlations, we use the respective logarithmic errors bars, $\sigma_{L_{X}^{*}}$ and $\sigma_{T_{a}^{*}}$, to formally define a fit -error parameter $u \equiv \sqrt{\sigma_{L_{X}^{*}}^{2}+\sigma_{T_{a}^{*}}^{2}}$. This definition is used to distinguish the canonical shaped light curves from the more irregular ones, perturbed by "secondary" flares and various non-uniformities. (For the discussion of systematics issues regarding the choise of systematics see [14], while for general discussion on systematics for luminosity relations see [11]). The symmetric error bars quoted are computed with the method of [16] that takes into account the hidden errors and thus gives greater error estimates than the ones obtained with the Marquardt Levemberg algorithm [24].

Our analyzed sample of 77 GRBs from the redshift range $0.08-8.26$ includes afterglows of 66 long GRBs and 11 GRBs whose nature is debated, the IC between long and short GRBs described by [32] as an apparent (sub)class of bursts with a short initial pulse followed by an extended lowintensity emission phase. Our long GRB sample also includes eight X -ray flashes (XRFs; 060108, 051016B, 050315, 050319 [20], 050401, 050416A, 060512, 080330 [37]). XRFs are scattered within the long GRB distribution in Figure 1, providing further support to a hypothesis that both these phenomena have the same progenitors [27]. To study physically homogeneous samples, we decided here to analyze the sub-samples of 66 long GRBs (including XRFs).

Regarding the investigation of the correlations of $L_{a}^{*}$ with several other prompt emission parameters we can estimate the characteristic luminosity of a burst using different characteristic times, $T_{45}, T_{90}$ and $T_{p}$, where $T_{45}$ is the time spanned by the brightest 45 per cent of the total counts above the background [34] and $T_{p}$ is the fitted transition time in which the exponential decay in the prompt phase changes to a power law decay. Here we define $<L_{p}^{*}>_{45} \equiv E_{i s o} / T_{45}^{*},<L_{p}^{*}>_{90} \equiv E_{i s o} / T_{90}^{*}$ and $<L_{p}^{*}>_{T p} \equiv E_{i s o} / T_{p}^{*}$ and we have analyzed correlations between logarithms of the prompt emission parameters $E_{i s o},<L_{p}^{*}>_{45},<L_{p}^{*}>_{90},<L_{p}^{*}>_{T p}$, and the parameters $L_{a}^{*}$ characterizing the afterglow light curve. The $E_{i s o}, T_{90}$ and $T_{45}$ values are listed in $[4,6]$, while $T_{p}$ is the one used to fit the Willingale model. 

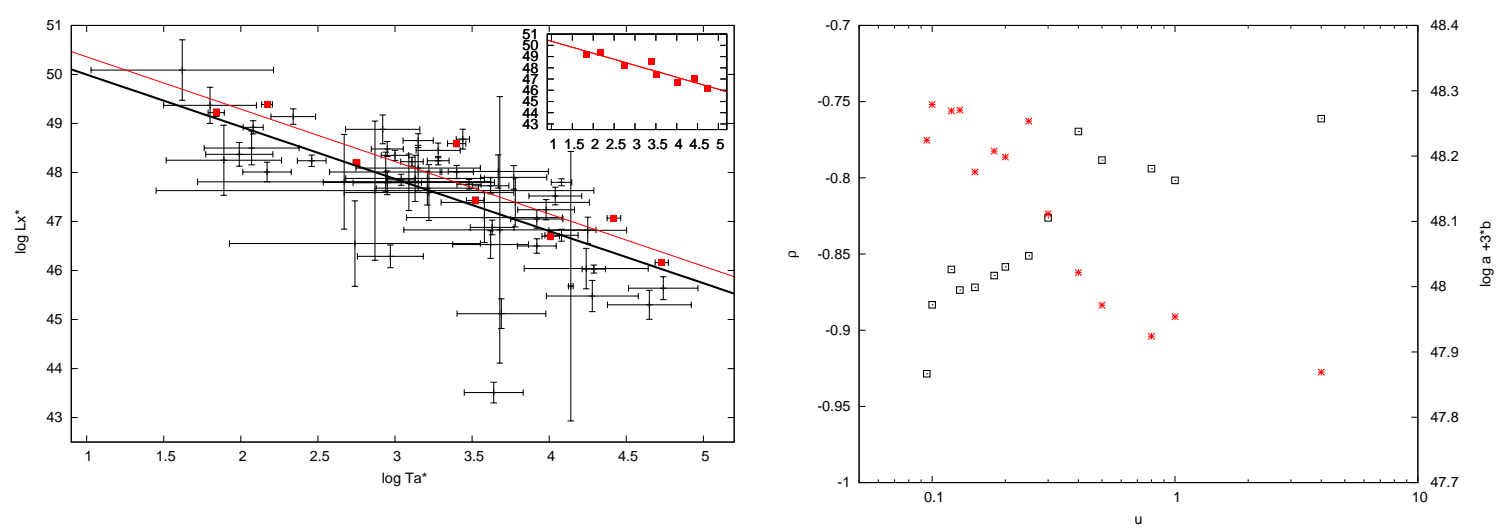

Figure 1: Left panel: $L_{X}^{*}$ vs $T_{a}^{*}$ distribution for the sample of 62 long afterglows with $u<4$, with the fitted correlation line in black. The upper red line, fitted to the 8 lowest error (red) points, forms approximately an upper envelope of the full distribution. The upper envelope points with the fitted line are separately presented in an inset panel. On the right panel, Left vertical axis: correlation coefficients $\rho\left(\log L_{X}^{*}, \log T_{a}^{*}\right)$ vs the error parameter $u$ for the long GRBs are presented with black squares. The right vertical axis: normalizations of the fitted correlation lines at $\log T_{a}^{*}=3.0 \mathrm{vs} u$ are presented with red asterisks.

\section{The results}

The obtained " $L_{X}^{*}$ versus $T_{a}^{*}$ " distributions for long GRBs (Figure $1,{ }^{2}$ ) clearly demonstrate the existence of significant LT correlations, characterized by the Spearman correlation coefficient, $\rho$, a non-parametric measure of statistical dependence between two variables [40]. From a visual inspection of Figure 1 and the analysis discussed later in Figure 1 right panel one can note that the lowest error events concentrate in the upper part of the distribution, forming a highly correlated subsample of the full distribution. To visualize this effect we decided to select eight points with smallest errors to define our limiting upper envelope subsample, $u<0.095$, see the inset panel in Figure 1.

For the full sample of 66 long GRBs one obtains $\rho_{L T} \equiv \rho\left(\log L_{X}^{*}, \log T_{a}^{*}\right)=-0.68$ and a probability of occurring of such correlation by chance within the uncorrelated sample $P=7.60 \times$ $10^{-9}$ (cf. [2]). If we remove a few large error points by imposing a constraint $u<4$, we have a limited sample of 62 long GRBs presented in Figure1, with $\rho_{L T}=-0.76, P=1.85 \times 10^{-11}$, and the fitted correlation line parameters $\log a=51.06 \pm 1.02$ and $b=-1.06_{-0.28}^{+0.27}$, while for the upper envelope sample we obtain, respectively, $\rho_{L T}=-0.93, P=1.7 \times 10^{-2}, \log a=51.39 \pm 0.90$ and $b=-1.05_{-0.20}^{+0.19}$.

To study the fit error systematic of GRB afterglows we show below, in Fig 1 on right panel, how the limiting upper value for $u$ in the analyzed sample, i.e. how selecting the afterglows with increasing precision of $L_{X}^{*}$ and $T_{a}^{*}$ fits, influences the LT correlation. We present changes of the $\rho_{L T}$ converging -with decreasing $u$-toward a nearly linear LT relation, as observed for our upper envelope sample. In the figure, e.g., we have 62 long GRBs for $u=4,33$ GRBs for $u=\overline{0.3,19 \text { GRBs }}$ for $u=0.15,13$ GRBs for $u=0.12$ and eight GRBs left for our limiting $u=0.095$. A presented accompanying systematic shift upward of the fitted correlation - as measured in the middle of the

\footnotetext{
${ }^{2}$ See the data table for all long and IC GRBs at http://www.oa.uj.edu.pl/M.Dainotti/GRB2010
} 


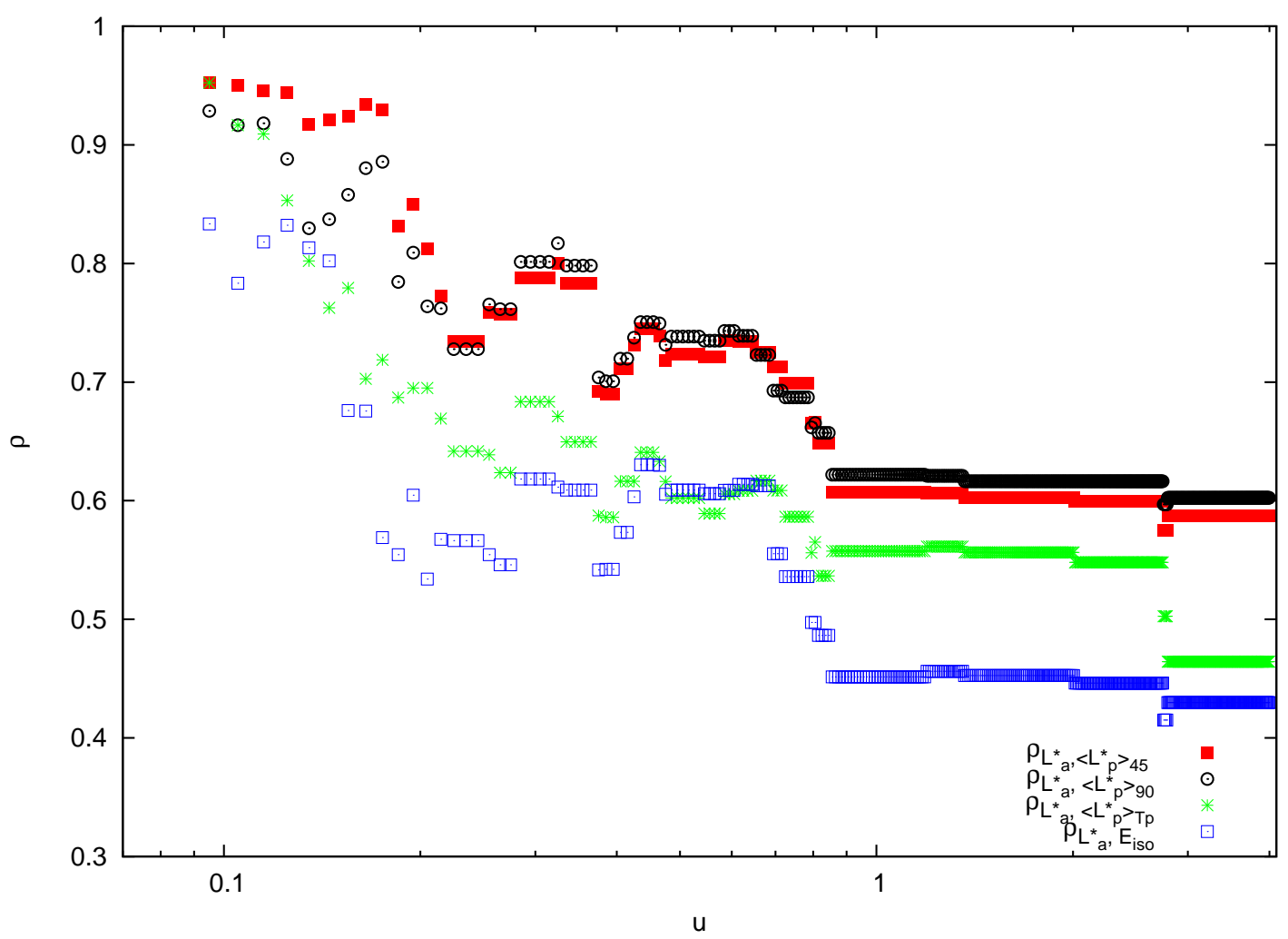

Figure 2: Correlation coefficients $\rho$ for the distributions $\log L_{a}^{*}-\log <L_{p}^{*}>_{45}$ (red squares), $\log L_{a}^{*}-\log <$ $L_{p}^{*}>_{90}$ (black circles), $\log L_{a}^{*}-\log <L_{p}^{*}>_{T p}$ (green asterixes) and $\log L_{a}^{*}-\log E_{i s o}$ (blue squares) for the long GRB subsamples with the varying maximum error parameter $u$.

distribution as $\log a-3.0 \cdot b$ (the fitted correlation line at selected $\left.\log T_{a}^{*}=3.0\right)$-with decreasing $u$, proves that the limiting $u \ll 1$ subsample forms the brightest afterglows in the LT distribution. This regular trend allows us to conclude that the subclass of all long GRBs with "canonical" afterglows forms a well defined physical class of sources exhibiting high correlation of their afterglow parameters. Presence of GRBs with light curves deviating from the [43] model increases the scatter in the LT distribution, with larger error points distributed preferentially below the small error ones. Let us also note that our limiting upper envelope subsample includes GRBs with redshifts reaching the maximum value of 'only' 2.75 , while the most distant GRB with $z=8.26$ disappears from the analyzed sample after decreasing $u$ below 0.25 .

We have represented changes of the $\rho_{L T}$ converging -with decreasing $u$ for the $\log L_{a}^{*}-\log <$ $L_{p}^{*}>_{45}$ and the other distributions considered in this study, involving $E_{i s o},<L_{p}^{*}>_{90}$ and $\left\langle L_{p}^{*}>_{T p}\right.$. The highest correlated sample is represented by the $\log L_{a}^{*}-\log <L_{p}^{*}>_{45}$, but also the other distributions show significant correlations, with the lowest $u$ events forming in all cases tightly correlated subsamples of the full distribution (Fig. 2).

\section{Summary}

In this analysis we present the updtodate of the $L_{a}^{*}-T_{a}^{*}(\mathrm{LT})$ correlation and new signifi- 
cant correlations between the luminosity of the afterglow plateau phase, $L_{a}^{*}$, and parameters of the prompt emission, including the mean luminosities and the integral energy derived for this emission. For the light curves which are smooth and well fitted by the [43] phenomenological model we find tight correlations in the analyzed distributions, showing that only GRBs with regular light curves exhibit strict physical scalings between their observed characteristics. Thus only such events can be considered to form the standard GRB sample, to be used for both GRB detailed physical model discussion and, possibly, to work out the GRB-related cosmological standard candle. A progress in both issues requires to increase an observed number of the canonical light curve GRBs, not by simply increasing the total number of GRBs with know redshifts. GRBs with the light curve nonuniformities exhibit weaker correlations of the plateau phase and the prompt emission energetics.

\section{Acknowledgments}

This work made use of data supplied by the UK Swift Science Data Center at the University of Leicester. MGD and MO are grateful for support from the Polish Ministry of Science and Higher Education through the grant N N203 380336.

\section{References}

[1] Amati, L., F. Frontera, Tavani, M., in 't Zand J. J. M., Antonelli, A. et al. 2002, A\&A, 390, 81 Ű89

[2] Bevington, P. R., \& Robinson, D. K. 2003, Data Reduction and Error Analysis for the Physical Sciences (3rd ed.; New York: McGraw-Hill)

[3] Bloom, J. S., Frail, D. A., Sari, 2001, ApJ, 121, 2879

[4] Butler, N. R., et al. 2007, ApJ, 671, 656

[5] Butler, N. R., Bloom, J. S., Poznanski, D. 2009, ApJ, 694, 76B

[6] Butler, N. R., Bloom, J. S. \& Poznanski, D. 2010, ApJ, 711, 495B

[7] Cannizzo,J. K. \& Gehrels, N. 2009, ApJ, 700, 1047

[8] Cabrera, J. I., et al. 2009, MNRAS, 382, 342

[9] Cardone, V.F, Capozziello, S. \& Dainotti, M. G. 2009, MNRAS, 400, 775

[10] Cardone, V.F, Dainotti, M.G., Capozziello, S. \& Willingale, R. 2010 MNRAS, 400, 775

[11] Collazzi, A.C., Schaefer, B.E., \& Moree, J. A. 2011 to appear on APJ arXiv:1101.1285

[12] Dainotti, M. G., Cardone, V. F., \& Capozziello, S. 2008, MNRAS, 391, L79

[13] Dainotti, M. G., Willingale, R., Capozziello, S., Cardone, V.F., Ostrowski, M., 2010, ApJL, 722, L215

[14] Dainotti, M. G., Cardone, V.F., Capozziello, S., Ostrowski, M., Willingale, R., 2011, ApJ, 730, 135

[15] Dall'Osso et al. 2010, submitted to A\&A, arXiv:astroph 1004.2788

[16] D’ Agostini, G. arXiv:astro-ph/0511182 (2005)

[17] Evans, P. et al. MNRAS, 2009, 397, 1177

[18] Fenimore, E. E., \& Ramirez - Ruiz, E. 2000, ApJ, 539, 712 
[19] Firmani, C., Ghisellini, G., Avila-Reese, V., \& Ghirlanda, G. 2006, MNRAS, 370, 185

[20] Gendre, B, Galli, A. \& Piro, L. 2007, A\&A 465, L13

[21] Ghirlanda G., Ghisellini, G. \& Firmani, C., 2006, New J. Phys., 8, 123

[22] Ghirlanda, G., Ghisellini, G., \& Lazzati, D. 2004, ApJ, 616, 331

[23] Ghisellini, G., Nardini, M., Ghirlanda G., \& Celotti, A., MNRAS, 393, 253 (2009)

[24] Marquardt, D. 1963, SIAM J. Applied Phys., 11, 431

[25] Yamazaki, R. 2009, ApJ, 690, L118

[26] Izzo, L. Capozziello, S., Covone, G., Capaccioli, M. 2009, A\& A, 508, 63I

[27] Ioka, K. \& Nakamura, T. 2001, A\&A, 554, L163

[28] Liang, E., \& Zhang, B. 2005, ApJ, 633, 611

[29] Liang, E. W., \& Zhang, B. 2006, MNRAS, 369, L37

[30] Liang, N., Wu, P. \& Zhang, S. N 2009, PhRvD., 81h, 3518L2

[31] Norris, J. P., Marani, G. F., \& Bonnell, J. T. 2001, ApJ, 534, 248

[32] Norris, J. P. \& Bonnell, J.T. 2006, A\&A, 643, 266

[33] Nousek, J. A, et al. 2006, A\&A, 642, 389

[34] Riechart, D.E., Lamb, D.Q., Fenimore, E.E., Ramirez - Ruiz, E., Cline, T.L. 2001, ApJ, 552, 57

[35] Riess, A. G et al., 2007, ApJ, 659, 98

[36] Salvaterra, R., et al. 2009, Nature, 461, 1258S

[37] Sakamoto T., 2008, in AIP Conf. Proc., NANJING GAMMA-RAY BURST CONFERENCE, China, $1065,9 \mathrm{~S}$

[38] Shahmoradi, A. \& Nemiroff R. J. 2009, AIPC. 1133, 425S

[39] Schaefer, B.E. 2007, ApJ, 660, 16

[40] Spearman, C. 1904, The Am. J. Psychol, 15, 72

[41] Qi, S., Lu, T., \& Wang, F.-Y., 2009, MNRAS, 398, L78

[42] Tanvir, N. R. at al. 2009, Nature 461, 7268

[43] Willingale, R. W. et al., 2007, ApJ, 662, 1093

[44] Yu, B., Qi, S., \& Lu, T. 2009, ApJ, 705, L15 